\title{
Electrophysiological and behavioral correlates of arousal
}

\author{
RHAWN JOSEPH \\ UHS/The Chicago Medical School, North Chicago, Illinois 60064
}

and

\section{NANCY M. FORREST, DENISE FIDUCIA, PETER COMO, and JEROME SIEGEL University of Delaware, Newark, Delaware 19711}

\begin{abstract}
The relationships among levels of activity, responsiveness, exploration, and cortical arousal were assessed in a group of similarly reared male rats from a strain that was highly inbred to maximize genetic homogeneity. In Experiment 1, 22 subjects were tested in a complex, compartmentalized open field (i.e., a closed field), and those falling approximately $1 \mathrm{SD}$ above or below the group activity mean (eight were eliminated) were designated as high- or low-active, implanted with electrodes, and tested electrophysiologically to ascertain the level of cortical arousal as determined via measurements of visually evoked potential afterdischarge amplitude. High-active subjects demonstrated a significantly lower afterdischarge amplitude, indicating that behavioral arousal may be indexed by a readily quantifiable electrophysiological measure. A second group of 14 rats (Experiment 2) was tested and retested after 45 days in the closed field, establishing that high- or low-behavioral responsiveness is a stable characteristic. Subjects then received 2 days' exposure to one of two dissimilar compartments of an exploratory apparatus and were tested to determine amount of time spent in the familiar vs. novel compartments. High-active rats were found to demonstrate significantly shorter initial response latencies. However, no other differences were found. These rats were next tested in a completely open field. No significant activity differences were discovered. These findings show that a high level of arousal as a response to a highly complex environment or intense stimulation is not related to generalized activity elicited in a low-complexity environment (e.g., the open field). These findings also indicate that similarly reared rats may be differently influenced by novel, complex, or intense stimuli, such that some subjects become highly responsive and cortically aroused, whereas others respond with low levels of behavioral and cortical activation. The findings are discussed in terms of cortical and subcortical mechanisms mediating arousal.
\end{abstract}

The factors contributing to individual differences in activity, exploration, and cortical arousal are not clearly understood. Behavioral, hormonal, electrophysiological, and neuroanatomical studies of a variety of species have suggested that differences in individual responsiveness are linked to differential rearing conditions, gender, genetic factors, and structural or functional differences in the excitability of the nervous system (Buchsbaum, 1974; Como, Joseph, Fiducia, Siegel, \& Lukas, 1979; Fleming, Rhodes, Wilson, \& Shearer, 1973; Joseph, 1979; Joseph \& Casagrande, 1978, 1980; Joseph \& Gallagher, 1980; Joseph, Hess, \& Birecree, 1978; Konrad \&

This research was partially supported by Army Research Office Contract No. DA AG 29-80-K-0015 to J.S. while the first author was associated with the Department of Psychology and the Institute for Neuroscience, University of Delaware, Newark, Delaware 19711. Address reprint requests to : Rhawn Joseph, Department of Psychology, UHS/The Chicago Medical School, North Chicago, Illinois 60064 .
Bagshaw, 1970; Lukas \& Siegel, 1977b; Zuckerman, Murtaugh, \& Siegel, 1974). The purpose of the present study was to assess the relationships among activity, behavioral responsiveness, and cortical arousal as measured by visually evoked potentials in a group of similarly reared, same-sex rats from a strain highly inbred to maximize genetic homogeneity.

In a number of studies, it has been demonstrated that the visually evoked potential (VEP) afterdischarge (AD) is a behaviorally sensitive form of EEG cortical synchrony that is dependent upon recurrent excitatoryinhibitory activity in the dorsal lateral geniculate nucleus and modulating influences of the thalamus, limbic system, and reticular activating system (RAS) (Bigler, 1975; Fleming et al., 1973; Klingbern, 1971; Knispel \& Siegel, 1972; Lukas \& Siegel, 1977a, 1977b; Schwartzbaum, 1975). For example, animals in a state of relaxed wakefulness (Klingbern, 1971) or rat strains that respond with low levels of behavioral activation (Fleming et al., 1973) show cortical synchronous EEG activity, as well as high-amplitude 
VEP ADs (Shearer \& Creel, 1978), whereas rat strains that are characteristically highly active have a comparatively lower amplitude low-voltage fast EEG. Moreover, low-voltage fast EEG produced by activation of the RAS greatly dampens AD amplitude, whereas the $A D$ is optimally produced during low levels of RAS activity (Fleming et al., 1973; Schwartzbaum, 1975). These findings indicate, therefore, that the amplitude of the VEP AD may be viewed as an index of cortical and behavioral arousal.

To establish whether individual differences in behavioral responsiveness of an otherwise homogeneous population of rats are related to cortical arousal (Experiment 1), subjects were tested in a complex closed-field apparatus (Joseph \& Gallagher, 1980), were classified as high- or low-active, and were implanted with chronic indwelling electrodes, and the relationship between behavior and VEP AD amplitude was ascertained. To establish whether behavioral responsiveness is a stable trait (Experiment 2), a second group of subjects were tested, then retested after $\mathbf{4 5}$ days in the closed field. To determine whether behavioral responsiveness is an interactive function of the individual and environment, or a stable characteristic expressed similarly across environments regardless of stimulus intensity, novelty, or complexity, these subjects were also tested in an apparatus designed to assess exploratory behavior and in a neutral, lower complexity, open-field environment.

\section{EXPERIMENT 1}

\section{Method}

Subjects. Twenty-two male Delaware-Wistar rats born to six females bred in this laboratory were weaned at 25 days of age, placed in two large $(48.5 \times 40 \times 20.5 \mathrm{~cm})$ standard-rack suspended cages for 20 days, then randomly separated into four large rearing cages (five to six subjects in each) until 70 days of age, at which time they were placed individually in standard-rack cages $(40 \times 23 \times$ $20.5 \mathrm{~cm}$ ). At the onset of the behavioral and electrophysiological tests, the subjects were 90 and 120 days of age, respectively. All the subjects were maintained on ad-lib food and water in a colony room with a reversed 12 -h light/dark cycle and were tested during the dark phase.

Apparatus. To measure activity, a compartmentalized open field, referred to as a closed field and similar to that described by Joseph and Gallagher (1980), was employed. Briefly, the apparatus $(93 \times 82.5 \mathrm{~cm})$ ronsisted of one startbox $(43 \times 18.5 \mathrm{~cm})$, a $2-\mathrm{cm}$ wire-mesh floor, marked into $10.5-\mathrm{cm}$ squares, and mazelike alleyways of wooden barrier board. Illumination during behavioral tests was provided by a single $15-\mathrm{W}$ red light suspended $2 \mathrm{~m}$ directly above the field, and extraneous sound was masked with 85-90-dB white noise (BRS/LVE audio generator).

For conducting the electrophysiological study, the subjects were tested in a small $(60 \times 40 \mathrm{~cm})$ chamber lined with reflecting aluminum panels. The visual potential was elicited by a Grass PSI photostimulator flashtube enclosed in a sound-insulated wooden box with a frosted $.5-\mathrm{cm}$ Plexiglas cover on the front to provide even diffusion of light. The flashtube was placed in the chamber, $15 \mathrm{~cm}$ away from the subject. VEPs were amplified with a Grass P511 preamplifier, recorded on a Sanborn FM tape recorder (Model 3917B), and averaged by a Nuclear Chicago data retrieval computer (Model 7100), and the VEP averages were written out on a Mosley X-Y plotter (Model 7590).

Procedure. Prior to the onset of behavioral testing, all the subjects were briefly handled for four consecutive days to reduce cage-emergence stress and handling influences (Joseph, 1979). The subjects were habituated to the testing room $10 \mathrm{~min}$ before each test and were placed in a startbox, and their emergence latencies and square crossings were tabulated according to previously described criteria (Joseph et al., 1978). The subjects were tested for $10 \mathrm{~min} /$ day for four consecutive days. Observers were hidden from view throughout. The subjects were distinguished as being either high- or low-active, depending on their tendency to consistently fall $1 \mathrm{SD}$ above or below the daily square-crossing activity means, Eight rats were then eliminated, and the remaining subjects were coded so that all further assessment proceeded blindly.

The subjects were anesthetized with injections of Ketaset and chloral hydrate and were implanted with stainless steel screw electrodes, placed $2 \mathrm{~mm}$ anterior to lambda and $3 \mathrm{~mm}$ lateral to the midline (Area 17), $3 \mathrm{~mm}$ posterior to lambda (cerebellar ground electrode), and $7 \mathrm{~mm}$ anterior to bregma (reference electrode). All the subjects were permitted 7 days of recovery.

Evoked potential analysis. All rats were wrapped in a cloth harness and restrained, leaving only their heads exposed. After approximately $3 \mathrm{~min}$ of habituation, the subjects were placed in the testing chamber directly facing the flash unit. To assess for group differences in AD amplitude as a function of stimulus intensity, five intensities of light flash were each presented 30 times at a rate of $1 \mathrm{flash} / 5 \mathrm{sec}$, with $20 \mathrm{sec}$ elapsing before the onset of each intensity series. The intensities in foot candles were 5,17 , 55,155 , and 370 (as measured by an Alphametrics 1010 photometer placed $15 \mathrm{~cm}$ in front of the flash unit). Order of intensity presentation was determined randomly and held constant for all the subjects. The amplitude of the first peak of the AD was computed and averaged for each of the five intensities, using 400 data points over a 500-msec analysis interval. The first component of the AD was found to fall approximately $135 \mathrm{msec}$ (P3-N3) after sweep onset (see Figure 1B). Similar findings have been reported eisewhere (Como et al., 1979; Shearer \& Creel, 1978).

\section{Results}

Closed-field activity. A 2 by 4 analysis of variance (ANOVA) was performed on group scores (high- vs.
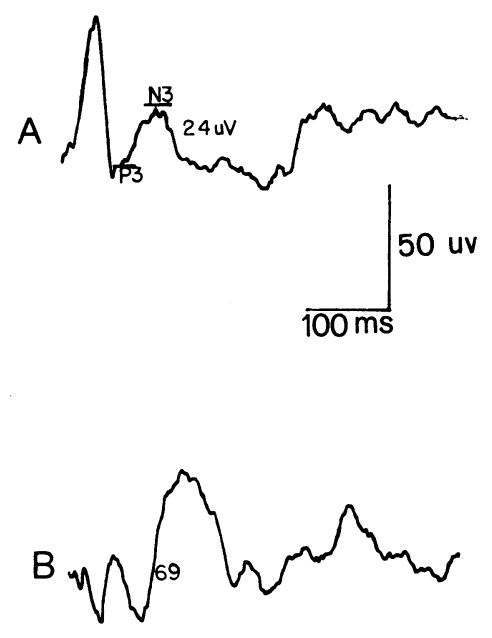

Figure 1. The averaged EP and AD of high-active Subject 1 (A) and low-active Subject 12 (B) in response to 30 sweeps of the second-intensity light flash. Note that the first peak of the AD (P3-N3) is much lower in the high-active rat. 
Table 1

Closed-Field Behavior and Afterdischarge Amplitude (in Microvolts) Across Five Intensities

\begin{tabular}{ccccccc}
\hline & & \multicolumn{5}{c}{ Flash Intensity } \\
\cline { 3 - 7 } Subject & $\begin{array}{c}\text { Closed-Field } \\
\text { Mean }\end{array}$ & 1 & 2 & 3 & 4 & 5 \\
\hline & & \multicolumn{5}{c}{ High-Active Group } \\
Mean & 275.8 & 23.22 & 28.58 & 19.91 & 15.88 & 18.18 \\
1 & 303.2 & 27.78 & 23.62 & 11.12 & 36.12 & 16.67 \\
2 & 240.8 & 22.23 & 16.67 & 11.57 & 16.67 & 13.89 \\
3 & 358.0 & 19.45 & 11.12 & 16.67 & 13.89 & 36.12 \\
4 & 272.3 & 15.27 & 62.50 & 29.17 & 5.56 & 19.44 \\
14 & 247.0 & 8.33 & 13.89 & 11.12 & 11.12 & 12.50 \\
15 & 245.3 & 52.78 & 66.67 & 45.83 & 5.56 & 13.33 \\
17 & 263.8 & 16.67 & 5.56 & 13.89 & 22.23 & 15.28 \\
& & & Low-Active Group & & \\
Mean & 138.4 & 47.23 & 57.94 & 40.87 & 44.05 & 36.11 \\
5 & 145.7 & 13.89 & 36.11 & 51.39 & 45.83 & 44.44 \\
7 & 78.0 & 20.84 & 36.12 & 41.67 & 47.23 & 27.78 \\
8 & 184.3 & 69.45 & 86.12 & 68.06 & 97.23 & 75.00 \\
9 & 125.0 & 41.67 & 33.34 & 27.78 & 30.56 & 27.78 \\
10 & 184.5 & 48.62 & 58.34 & 52.78 & 50.00 & 47.23 \\
12 & 65.0 & 83.33 & 69.44 & 19.44 & 16.67 & 11.11 \\
19 & 186.5 & 52.78 & 86.11 & 25.00 & 20.83 & 19.44 \\
\hline
\end{tabular}

low-active) across testing days, and it was established that the remaining 14 subjects demonstrated significantly different amounts of behavioral responsiveness in the closed field $[F(1,12)=28.34, p<.001]$.

VEP amplitudes. A clear and highly significant difference in AD amplitude between high- and lowactive rats was discovered $[F(1,12)=12.158, p<.004]$, demonstrating that the highly responsive subjects maintained significantly lower amplitudes across all intensities as compared with the behaviorally lowactive rats. Figure 1 illustrates a typical EP and $A D$ in a high-active rat (1A) and a low-active rat (1B) to the same intensity light flash.

Table 1 presents, for each rat, the mean activity score in the closed field across 4 days and the ampli-

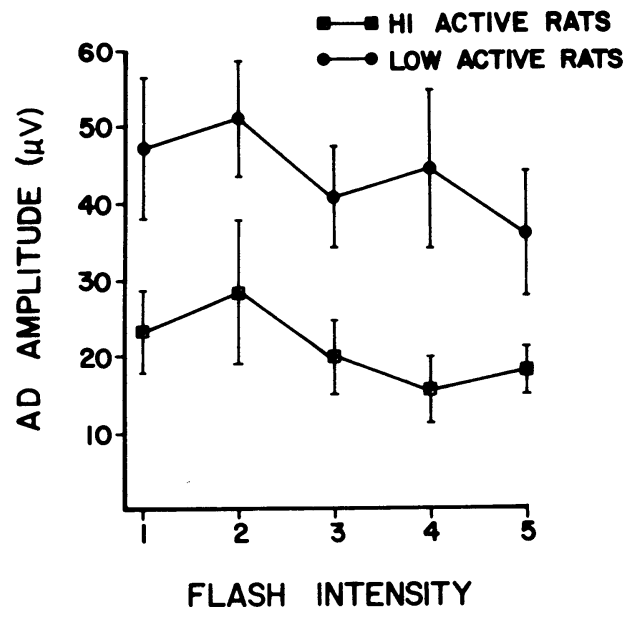

Figure 2. Group mean \pm the standard error of the AD amplitudes (P3-N3) of high- and low-active rats across five intensities of light flash. tude of the computer-averaged afterdischarge (P3-N3) for each of the five flash intensities.

The relationships of AD amplitudes and behavioral activity levels are graphically displayed in Figure 2. The slopes of $A D$ amplitudes for both the high- and low-active rats show a trend toward reduction as a function of flash intensity, but these slopes are statistically nonsignificant. It may be that this trend is due to an arousal effect with high-intensity light flashes or is related to EP reducing, which we have reported in cats (Lukas \& Siegel, 1977b).

\section{Discussion}

The results clearly demonstrate that individual and group differences in behavioral responsiveness to complex, novel, and intense forms of stimuli are correlated with cortical activation, as indicated by the amplitude of the VEP afterdischarge. Rats responding with high levels of behavioral activation exhibit low-amplitude ADs when stimulated with various intensities of light flashes, whereas rats demonstrating low behavioral responsiveness respond to light-flash stimulation with high-amplitude ADs and thus demonstrate lower levels of cortical activation. These findings indicate that low-active subjects possibly have a lower threshold for activation of cortical inhibition, as mediated via ascending reticular influences (Fuster, 1961; Orem \& Feeney, 1971) or through intracortical activity (Dell, Bonvallet, \& Hugelin, 1961; Demetrescu, Demetrescu, \& Iosif, 1965; Steriade, 1968).

Although it could be argued that high-active rats are in fact responding with significantly higher levels of exploratory behavior, and the VEP AD components are actually measuring cortical processing of environmental cues, it must be cautioned that 
the behavioral factors measured were dependent upon activity and responsiveness to complex and intense forms of stimulation, variables that are often only weakly linked to exploratory behavior (Joseph \& Gallagher, 1980). As pointed out by Joseph and Gallagher, such behavior is often indicative of tendencies to overrespond. It is noteworthy that, once aroused, neither the high- nor low-active subjects significantly increased their cortical arousal level when they were exposed to the intense light flashes, suggesting that the low-active rats respond with significantly lower levels of cortical activation than do high-active subjects, regardless of the stimulus parameters. To clarify these issues, Experiment 2 was performed to determine if differential levels of behavioral activation in a closed-field environment reflect differences in novelty seeking (exploratory behavior) or simply differences in reactivity.

\section{EXPERIMENT 2}

\section{Method}

Subjects. Fourteen male Delaware-Wistar rats born to four females bred in this laboratory were weaned, placed in two large rearing cages at 25 days of age, and, at 100 days of age, were assigned individually to single standard-rack suspended cages. The subjects were approximately 180 days of age at the onset of testing. All rats were maintained on ad-lib food and water throughout the experiment in a colony room with a reversed 12-h light/ dark cycle and were tested during the dark phase.

Apparatus. To measure behavioral responsiveness, the closed field employed in Experiment 1 was used. To assess preferences for familiar surroundings or for novelty (exploratory behavior), an apparatus similar to that described by Turpin (1977) was employed. Briefly, the apparatus was a $76 \times 15 \times 25 \mathrm{~cm}$ two- chamber wooden box, partially divided in the center by a wooden partition, with a $2-\mathrm{cm}$ wire-mesh floor. The walls were flat gray, with either $2-\mathrm{cm}$ horizontal or vertical black stripes. The effect of environmental stimuli on activity level was further assessed by testing all the subjects in a complexity-free, neutral environment-simple open field $(78 \times 78 \times 34 \mathrm{~cm})$, the floor of which (2-cm wire mesh) was divided into $10.5-\mathrm{cm}$ squares.

Procedure. Behavioral responsiveness was measured in a manner identical to that described in Experiment 1, with the exception that, after the first 4-day test series, all the subjects were retested after 45 days for an additional 4-day period. To assess novelty seeking, the subjects were categorized as high- or low-active, based on their behavioral responsiveness scores, and were assigned randomly to a habituation condition in which they were placed into one-half of the exploratory apparatus (familiarity conditioning). The subjects were habituated twice a day $-15 \mathrm{~min} / \mathrm{ses}-$ sion, with $3 \mathrm{~h}$ between each-for 2 days prior to testing. The subjects were also habituated for $10 \mathrm{~min}$ immediately prior to testing, which comprised one 10-min session/day on two consecutive days. At the beginning of each test session, the subjects were placed at the center portion of the exploratory apparatus, and the latency to first movement was scored, the initial compartment preference was determined (entering either the familiar or novel compartment), separate shuttles between compartments were tabulated, and the amount of time spent in each compartment was computed.

Ten days after completion of the exploratory testing, the subjects were assessed for activity elicited in the open field for 4 days, $10 \mathrm{~min} / \mathrm{session}$. Open-field testing procedures were identical to those employed in the closed field.

All the subjects were tested during their dark cycle, using red-light illumination, except during preference (exploratory) testing, in which $30-\mathrm{W}$ white-light illumination was provided $2 \mathrm{~m}$ directly above the apparatus. White noise $(80-90 \mathrm{~dB})$ was employed to mask extraneous sound throughout.

\section{Results}

The findings of this experiment are summarized in Table 2.

Closed-field activity. A 2 by 2 by 4 ANOVA was

Table 2

Closed- and Open-Field Activity of High- and Low-Active Rats and Total Time (in Seconds) Spent in Novel and Familiar Compartments of the Exploratory Apparatus Across Testing Days

\begin{tabular}{|c|c|c|c|c|c|c|c|}
\hline \multirow[b]{3}{*}{ Subject } & & & \multicolumn{4}{|c|}{ Exploration* } & \multirow{3}{*}{$\begin{array}{c}\text { Open-Field } \\
\text { Mean }\end{array}$} \\
\hline & \multicolumn{2}{|c|}{ Closed-Field Mean } & \multicolumn{2}{|c|}{ Novel } & \multicolumn{2}{|c|}{ Familiar } & \\
\hline & Test Series 1 & Test Series 2 & 1 & 2 & 1 & 2 & \\
\hline & \multicolumn{7}{|c|}{ High-Active Group } \\
\hline Mean & 309.0 & 260.3 & 300.1 & 273.9 & 299.9 & 326.1 & 228.8 \\
\hline 3 & 296.5 & 279.3 & 387.0 & 5.0 & 213.0 & 595.0 & 204.0 \\
\hline 6 & 326.0 & 286.8 & 557.0 & 506.0 & 43.0 & 94.0 & 246.0 \\
\hline 8 & 275.5 & 311.5 & 247.0 & 364.0 & 353.0 & 236.0 & 260.0 \\
\hline 11 & 331.8 & 315.0 & 122.0 & 97.0 & 478.0 & 503.0 & 219.8 \\
\hline 14 & 375.8 & 259.5 & 78.0 & 227.0 & 522.0 & 373.0 & 253.8 \\
\hline 15 & 248.0 & 227.5 & 539.0 & 517.0 & 61.0 & 83.0 & 187.5 \\
\hline \multirow[t]{2}{*}{16} & 309.5 & 142.5 & 171.0 & 201.0 & 429.0 & 399.0 & 230.5 \\
\hline & \multicolumn{7}{|c|}{ Low-Active Group } \\
\hline Mean & 185.1 & 197.0 & 252.3 & 352.0 & 347.7 & 248.0 & 198.0 \\
\hline 2 & 160.8 & 168.8 & 521.0 & 597.0 & 79.0 & 3.0 & 121.5 \\
\hline 4 & 207.8 & 142.5 & 168.0 & 247.0 & 432.0 & 353.0 & 158.8 \\
\hline 7 & 230.0 & 237.5 & 7.0 & 173.0 & 593.0 & 427.0 & 211.0 \\
\hline 9 & 204.5 & 181.5 & 327.0 & 442.0 & 273.0 & 158.0 & 155.0 \\
\hline 10 & 132.3 & 232.5 & 69.0 & 71.0 & 531.0 & 529.0 & 218.0 \\
\hline 12 & 183.5 & 171.5 & 445.0 & 405.0 & 155.0 & 195.0 & 296.0 \\
\hline 13 & 177.0 & 244.5 & 229.0 & 529.0 & 371.0 & 71.0 & 225.3 \\
\hline
\end{tabular}

*600 sec maximum per test day. 
computed for groups (high- vs. low-active), test series (first 4 days vs. last 4 ), and closed-field behavior across all testing days. As in Experiment 1, the activity differences between groups were highly significant and were maintained across the four test sessions $[\mathrm{F}(1,12)=30.149, \mathrm{p}<.001]$, as well as during the first $[F(1,12)=38.79, p<.001]$ and second tests $[F(1,12)=5.36, p<.05]$.

Novelty seeking. A 2 by 2 by 2 ANOVA was performed across high- and low-active groups, habituation conditions, and the following dependent variables: movement latency, initial preference, shuttles between compartments, and total time spent in each. A significant main effect was found for movement latency across testing days $[\mathrm{F}(1,12)=5.05, \mathrm{p}<.05]$, such that high-active rats demonstrated a shorter response latency than did low-active subjects. No other significant differences were found.

Open-field activity. A 2 by 4 ANOVA was performed on all open-field behavior. No significant differences were found between groups.

\section{Discussion}

As demonstrated in Experiment 2, tendencies to respond with high levels of behavioral activation and arousal are dependent upon the stimulus parameters of the testing environment (e.g., complexity, intensity, novelty). Hence, in a neutral, relatively low-arousal environment, the behavior of high- and low-active rats is indistinguishable. However, these same subjects, when placed in the more complex closed field (compartmentalized open field), responded with high or low levels of behavioral activity and continued to do so when retested after 45 days. Although it could be argued that high-active rats are comparatively more exploratory, it is curious that there were no signs of habituation when they were retested in the closed field. Moreover, when provided with the opportunity to "explore" a novel compartment (vs. a familiar environment), no group differences were found. Since the stimulus parameters of the "exploratory" apparatus differed only in regard to horizontal or vertical position of wall stripes, it is likely that the compartment was not complex enough to elicit differential responding. Nevertheless, it is interesting to note that high-active rats were initially more responsive, demonstrating the quickest movement latencies when first placed in the center of the exploratory apparatus.

The significant difference in response latency, the failure to find activity differences in the open field, and the behavioral responsiveness that results from placement in a highly novel, complex environment suggests that high-active rats, although not constantly functioning at high levels of arousal, tend to become highly aroused and overresponsive when exposed to complex and intense forms of stimulation.
The behavioral and electrophysiological results lend themselves to the suggestion that this tendency to overrespond may be due to a failure to initiate cortical inhibition of ascending reticular and thalamic activity or to possible differences in cortical-subcortical structure and function, particularly in the frontal cortex region.

These suggestions are in part supported by the similarities of the behavior of high-active rats to the behavior of rats with frontal pole lesions (Como et al., 1980) and to animals reared in an impoverished environment (Joseph \& Gallagher, 1980; Konrad \& Bagshaw, 1970). Interestingly, impoverished rearing conditions have been shown to decrease dendritic density in the frontal regions (Walsh, Cummins, Budtz-Olsen, \& Torok, 1972). This is significant in that the frontal cortex mediates motor output (Luria, 1973), exerts inhibitory influences on the reticular activating system (Lineberry \& Siegel, 1971), receives terminal projections from the secondary association cortex of all sensory modalities (Jones \& Powell, 1970), and thus mediates and organizes behavior in response to the sensory environment (Luria, 1973; Nauta, 1972). Hence, high levels of arousal and overresponsiveness may result from a lowered stimulus threshold, as well as from a failure to integrate incoming sensory messages with motor output due to frontal-lobe dysfunction. In a complex environment, there is thus excessive cortical arousal, significant motor overflow, and, as demonstrated in deprived animals (Joseph \& Gallagher, 1980), possible learning deficits resulting from an inability to successfully integrate and selectively respond to complex environmental stimuli.

\section{REFERENCES}

Bigler, E. D. Lateral geniculate multiple-unit activity related to metrazol potentiated after-discharge. Electroencephalography and Clinical Neurophysiology, 1975, 39, 491-497.

Bigler, E. D. Neurophysiology, neuropharmacology and behavioral relationships of the visual system evoked afterdischarges: A review. Biobehavioral Reviews, 1977, 1, 95-112.

Buchsbaum, M. S. Average evoked responses and stimulus intensity in identical and fraternal twins. Physiological Psychology, 1974, 2, 365-370.

Como, P. G., Jose Ph, R., Fiducia, D., Siegel, J., \& Lukas, J. Visually evoked potentials and after-discharge as a function of arousal and frontal lesion in rats. Proceedings of the Society for Neuroscience, 1979, 5, 202.

Dell, P., Bonvallet, M., \& Hugelin, A. Mechanisms of reticular deactivation. In G. E. W. Wolstenholme \& M. O'Connor (Eds.), The nature of sleep. London: Churchill, 1961.

Demetrescu, M., Demetrescu, M., \& Iosif, G. The tonic control of cortical responsiveness by inhibitory and facilitatory diffuse influences. Electroencephalography and Clinical Neurophysiology, 1965, 18, 1-24.

Fleming, D. W., Rhodes, L. E., Wilson, C. F., \& Shearer, D. E. Strain differences in the elicitation of electrocortical after-discharges. Physiology \& Behavior, 1973, 10, 879-885.

Fuster, J. M. Excitation and inhibition of neuronal firing in 
visual cortex by reticular formation. Science, 1961, 133, 20012012.

Jones, E. G., \& Powell, T. P. S. An anatomical study of converging sensory pathways within the cerebral cortex of the monkey. Brain, 1970, 93, 793-820.

JOSEPH, R. Effects of rearing and sex on maze learning and competitive exploration. Journal of Psychology, 1979, 101, $37-43$.

Joseph, R., \& Casagrande, V. A. Visual field defects and morphological changes resulting from monocular deprivation in prosimian primates. Proceedings of the Society for Neuroscience, 1978, 4, 2021.

Joseph, R., \& Casagrande, V. A. Visual defects and recovery following lid closure in a prosimian primate. Behavioral Brain Research, 1980, 1, 201-223.

Joseph, R., \& Gallagher, R. E. Gender and early environmental influences on activity, overresponsiveness and exploration. Developmental Psychobiology, 1980, 13, 527-544.

Joseph, R., Hess, S., \& Birecree, E. Effects of hormone manipulations and exploration on sex differences in maze learning. Behavioral Biology, 1978, 23, 264-277.

KLINGBe RN, F. Hypersynchrony and learning. In G. Adam (Ed.), Biology of memory. New York: Plenum Press, 1971.

Knispel, J. D., \& Siegel, J. Tegmental stimulation: Aversive effects on behavior and modulation of visual evoked potentials. Brain Research, 1972, 37, 319-321.

Konrad, K. W., \& Bagshaw, M. Effects of novel stimuli on cats reared in a restricted environment. Journal of Comparative and Physiological Psychology, 1970, 70, 157-164.

Lineberry, C. G., \& Siegel, J. EEG synchronization, behavioral inhibition and mesencephalic unit effect produced by stimulation of orbital cortex, basal forebrain and caudate nucleus. Brain Research, 1971, 34, 143-161.

Lukas, J. H., \& SiEgel, J. Aversive noise effects on performance and thalamocortical responsiveness in cats. Physiology \& Behavior, 1977, 19, 555-559. (a)

Lukas, J. H., \& Siegel, J. Cortical mechanisms that augment or reduce evoked potentials in cats. Science, 1977, 198, 73-75. (b)

LuRIA, A. R. The working brain. New York: Basic Books, 1973.

Nauta, W. J. H. Neural associations of the frontal cortex. Acta Neurobiologica Experimentalis, 1972, 32, 125-140.

Orem, J., \& Feeney, D. M. Reciprocal reticular influences on cells in rostral and caudal visual cortex in the cat. Brain Research, 1971, 30, 200-203.

SchwartzBaUM, J. S. Interrelationships among multiunit activity of the midbrain reticular formation, and lateral geniculate nucleus, thalamocortical arousal and behavior in rats. Journal of Comparative and Physiological Psychology, 1975, 83, 131-157.

Shearer, D. E., \& Creel, D. The photically evoked afterdischarge: Current concepts and potential applications. Physiological Psychology, 1978, 6, 369-376.

STE RIADE, M. The flash evoked after-discharge. Brain Research, $1968,9,169-212$.

Turpin, B. Variations of early social experience and environmental preference in rats. Journal of Comparative and Physiological Psychology, 1977, 83, 131-157.

Walsh, R. N., Cummins, R. A., Budtz-Olsen, O. E., \& ToroK, A. Effects of environmental enrichment and deprivation on rat frontal cortex. International Journal of Neuroscience, 1972, 4, 239-242.

Zuckerman, M., Murtaugh, T. M., \& Siegel, J. Sensation seeking and cortical augmenting-reducing. Psychophysiology, 1974, 11, 535-542.

(Received for publication April 3, 1980; revision accepted November 20,1980 .) 